\title{
Relativistic second-order dissipative fluid dynamics at finite chemical potential
}

\author{
Amaresh Jaiswal ${ }^{1, a}$, Bengt Friman ${ }^{1}$, and Krzysztof Redlich ${ }^{2,3,4}$ \\ ${ }^{1}$ GSI, Helmholtzzentrum für Schwerionenforschung, Planckstrasse 1, D-64291 Darmstadt, Germany \\ ${ }^{2}$ Institute of Theoretical Physics, University of Wroclaw, PL-50204 Wroclaw, Poland \\ ${ }^{3}$ Extreme Matter Institute EMMI, GSI, Planckstrasse 1, D-64291 Darmstadt, Germany \\ ${ }^{4}$ Department of Physics, Duke University, Durham, North Carolina 27708, USA
}

\begin{abstract}
We employ a Chapman-Enskog like expansion for the distribution function close to equilibrium to solve the Boltzmann equation in the relaxation time approximation and subsequently derive second-order evolution equations for dissipative charge current and shear stress tensor for a system of massless quarks and gluons. We use quantum statistics for the phase space distribution functions to calculate the transport coefficients. We show that, the second-order evolution equations for the dissipative charge current and the shear stress tensor can be decoupled. We find that, for large chemical potential, the charge conductivity is small compared to the shear viscosity. Moreover, we demonstrate that the limiting behaviour of the ratio of heat conductivity to shear viscosity is identical to that obtained for a strongly coupled conformal plasma.
\end{abstract}

\section{Introduction}

At extreme conditions of temperature and density, quarks and gluons are deconfined to form quarkgluon plasma (QGP). The QGP behaves as a strongly coupled plasma having a very small shear viscosity-to-entropy density ratio, $\eta / s[1-7]$. Relativistic hydrodynamics has been quite successful in describing the space-time evolution of the QGP formed in relativistic heavy ion collisions and estimate its transport coefficients [8]. While most of the studies have focused on exploring the effects of shear and bulk viscosity on the QGP evolution, the dissipative charge current has been largely ignored. This may be due to the fact that at very high energies, baryon chemical potential are negligible. On the other hand, at lower energies such as those probed in the RHIC low-energy scan and at the upcoming Facility for Antiproton and Ion Research (FAIR), baryon chemical potential can no longer be ignored and therefore dissipative charge diffusion may play an important role.

Formulation of a causal theory of relativistic dissipative hydrodynamics are due to Grad [9], Müller [10] and Israel and Stewart (IS) [11]. However, the IS formulation of relativistic hydrodynamics from kinetic theory has several approximations and inconsistencies, the resolution of which is an active research area [12-25]. Here we present the derivation of causal (second-order) equations for dissipative charge current and shear stress tensor for a system of massless quarks and gluons. We employ an iterative Chapman-Enskog like expansion [26] to solve the Boltzmann equation in the

ae-mail: jaiswal.amaresh@gmail.com 
relaxation time approximation [18]. Subsequently, we derive evolution equations for the dissipative quantities and exact expressions for the associated transport coefficients. We employ quantum statistics for the quark and gluon phase-space distribution functions with a non-vanishing quark chemical potential. We show that the evolution equations for the dissipative charge current and the shear stress tensor can be decoupled. We find that, for large chemical potential, the charge conductivity is small compared to the shear viscosity. Finally we demonstrate that in the limiting cases, the ratio of heat conductivity to shear viscosity, obtained here in the relaxation-time approximation, is identical to that of a conformal fluid in the strong coupling limit.

\section{Relativistic hydrodynamics}

The conserved energy-momentum tensor and the net-quark current for a system of massless quarks and gluons can be expressed in terms of the phase-space distribution function as [27]

$$
\begin{aligned}
T^{\mu v} & =\int d p p^{\mu} p^{v}\left[g_{q}\left(f_{q}+f_{\bar{q}}\right)+g_{g} f_{g}\right]=\epsilon u^{\mu} u^{v}-P \Delta^{\mu v}+\pi^{\mu v}, \\
N^{\mu} & =\int d p p^{\mu}\left[g_{q}\left(f_{q}-f_{\bar{q}}\right)\right]=n u^{\mu}+n^{\mu},
\end{aligned}
$$

where $d p=d \mathbf{p} /\left[(2 \pi)^{3}|\mathbf{p}|\right], p^{\mu}$ is the particle four momenta, and $g_{q}$ and $g_{g}$ are the quark and gluon degeneracy factor, respectively. Here $f_{q}, f_{\bar{q}}$, and $f_{g}$ are the phase-space distribution functions for quarks, anti-quarks, and gluons. In the tensor decompositions, $\epsilon, P, n, \pi^{\mu \nu}$ and $n^{\mu}$ are the energy density, pressure, net quark number density, shear stress tensor and particle diffusion current, respectively. The bulk viscous pressure vanishes in the massless case. The projection operator $\Delta^{\mu \nu}=g^{\mu \nu}-u^{\mu} u^{\nu}$ is orthogonal to the hydrodynamic four-velocity $u^{\mu}$ defined in the Landau frame: $T^{\mu v} u_{v}=\epsilon u^{\mu}$. The conservation of energy-momentum, $\partial_{\mu} T^{\mu \nu}=0$, and particle four-current, $\partial_{\mu} N^{\mu}=0$, gives

$$
\begin{aligned}
\dot{\epsilon}+(\epsilon+P) \theta-\pi^{\mu v} \sigma_{\mu v} & =0, \\
(\epsilon+P) \dot{u}^{\alpha}-\nabla^{\alpha} P+\Delta_{v}^{\alpha} \partial_{\mu} \pi^{\mu v} & =0, \\
\dot{n}+n \theta+\partial_{\mu} n^{\mu} & =0 .
\end{aligned}
$$

Here we use the notation $\dot{A}=u^{\mu} \partial_{\mu} A$ for co-moving derivatives, $\theta \equiv \partial_{\mu} u^{\mu}$ for the expansion scalar, $\sigma^{\mu \nu} \equiv \frac{1}{2}\left(\nabla^{\mu} u^{v}+\nabla^{v} u^{\mu}\right)-\frac{1}{3} \theta \Delta^{\mu \nu}$ for the velocity stress tensor, and $\nabla^{\alpha}=\Delta^{\mu \alpha} \partial_{\mu}$ for space-like derivatives. by

The equilibrium phase-space distribution functions for quarks, anti-quarks and gluons are given

$$
f_{q}^{(0)}=\frac{1}{\exp (\beta u \cdot p-\alpha)+1}, \quad f_{\bar{q}}^{(0)}=\frac{1}{\exp (\beta u \cdot p+\alpha)+1}, \quad f_{g}^{(0)}=\frac{1}{\exp (\beta u \cdot p)-1},
$$

respectively, where $\beta=1 / T$ is the inverse temperature, $u \cdot p \equiv u_{\mu} p^{\mu}$ and $\alpha=\mu / T$ is the ratio of the quark chemical potential to temperature (we assume vanishing chemical potential for gluons). For a dissipative system, the temperature $T$ and chemical potential $\mu$ is determined by the matching condition $\epsilon=\epsilon_{0}$ and $n=n_{0}$. In equilibrium, the energy density, pressure and net quark number density for a system of massless quarks and gluons is given by

$$
\begin{aligned}
\epsilon_{0} & \equiv u_{\mu} u_{v} \int d p p^{\mu} p^{v}\left[g_{q}\left(f_{q}^{(0)}+f_{\bar{q}}^{(0)}\right)+g_{g} f_{g}^{(0)}\right]=\frac{\left(4 g_{g}+7 g_{q}\right) \pi^{2}}{120} T^{4}+\frac{g_{q}}{4} T^{2} \mu^{2}+\frac{g_{q}}{8 \pi^{2}} \mu^{4}, \\
P_{0} & \equiv-\frac{1}{3} \Delta_{\mu v} \int d p p^{\mu} p^{v}\left[g_{q}\left(f_{q}^{(0)}+f_{\bar{q}}^{(0)}\right)+g_{g} f_{g}^{(0)}\right]=\frac{\left(4 g_{g}+7 g_{q}\right) \pi^{2}}{360} T^{4}+\frac{g_{q}}{12} T^{2} \mu^{2}+\frac{g_{q}}{24 \pi^{2}} \mu^{4}, \\
n_{0} & \equiv u_{\mu} \int d p p^{\mu}\left[g_{q}\left(f_{q}^{(0)}-f_{\bar{q}}^{(0)}\right)\right]=\frac{g_{q}}{6} T^{2} \mu+\frac{g_{q}}{6 \pi^{2}} \mu^{3},
\end{aligned}
$$


Using the above equations and Eqs. (3)-(5), we obtain expressions for the derivatives of $\beta$ and $\alpha$ as

$$
\begin{aligned}
\dot{\beta} & =\frac{\beta}{3} \theta+O\left(\delta^{2}\right), \quad \dot{\alpha}=O\left(\delta^{2}\right), \\
\nabla^{\mu} \beta & =-\beta \dot{u}^{\mu}+\frac{n}{\epsilon+P} \nabla^{\mu} \alpha-\frac{\beta}{\epsilon+P} \Delta_{\rho}^{\mu} \partial_{\gamma} \pi^{\rho \gamma},
\end{aligned}
$$

where $O\left(\delta^{2}\right)$ represents terms which are of second order in derivatives. Note that while Eq. (11) is exact, Eq. (10) is terminated at first-order which is sufficient for the present work. For a system close to local thermodynamic equilibrium, $f=f^{(0)}+\delta f$, where $|\delta f| / f^{(0)} \ll 1$. Therefore, using Eqs. (1) and (2), the shear stress tensor $\pi^{\mu \nu}$ and the particle diffusion current $n^{\mu}$ can be written as

$$
\begin{aligned}
\pi^{\mu \nu} & =\Delta_{\alpha \beta}^{\mu \nu} \int d p p^{\alpha} p^{\beta}\left[g_{q}\left(\delta f_{q}+\delta f_{\bar{q}}\right)+g_{g} \delta f_{g}\right], \\
n^{\mu} & =\Delta_{\alpha}^{\mu} \int d p p^{\alpha}\left[g_{q}\left(\delta f_{q}-\delta f_{\bar{q}}\right)\right],
\end{aligned}
$$

where $\Delta_{\alpha \beta}^{\mu \nu} \equiv \frac{1}{2}\left(\Delta_{\alpha}^{\mu} \Delta_{\beta}^{v}+\Delta_{\beta}^{\mu} \Delta_{\alpha}^{v}\right)-\frac{1}{3} \Delta^{\mu v} \Delta_{\alpha \beta}$ is a traceless symmetric projection operator orthogonal to $u_{\mu}$ and $\Delta_{\mu \nu}$.

\section{Dissipative evolution equations}

The relativistic Boltzmann equation in the relaxation-time approximation is given by [28],

$$
p^{\mu} \partial_{\mu} f=-\frac{u \cdot p}{\tau_{R}}\left(f-f^{(0)}\right)
$$

where $\tau_{R}$ is the relaxation time. We employ iterative solution of the above equation to derive expressions for the dissipative quantities [17-19]. We obtain the first-order expressions for shear stress tensor and dissipative charge current as [18],

$$
\pi^{\mu v}=2 \beta_{\pi} \tau_{R} \sigma^{\mu \nu}, \quad n^{\mu}=\beta_{n} \tau_{R} \nabla^{\mu} \alpha .
$$

Here $\beta_{\pi}$ and $\beta_{n}$ are the first-order transport coefficients obtained as

$$
\beta_{\pi}=\frac{\epsilon+P}{5}, \quad \beta_{n}=\frac{J_{10}^{+}}{3}-\frac{n^{2} T}{\epsilon+P}
$$

where,

$$
J_{10}^{+} \equiv g_{q} \int d p(u \cdot p)\left(f_{q}^{(0)} \tilde{f}_{q}^{(0)}+f_{\bar{q}}^{(0)} \tilde{f}_{\bar{q}}^{(0)}\right)=\frac{g_{q}}{6} T^{3}+\frac{g_{q}}{2 \pi^{2}} T \mu^{2}=\frac{\pi^{2}+3 \alpha^{2}}{\alpha\left(\pi^{2}+\alpha^{2}\right)} n .
$$

Here $\tilde{f}^{(0)} \equiv 1-r f^{(0)}$, where $r=1$ for Fermions (quarks and anti-quarks) and $r=-1$ for Bosons (gluons).

To obtain the second-order dissipative equations, we consider the comoving derivative of Eqs. (12) and (13), and rewrite Eq. (14) in favour of $\delta \dot{f}$. Using first-order expressions for $\delta f$ obtained in Ref. [18] and performing the momentum integrations, we obtain the second-order evolution equations for $\pi^{\mu v}$ and $n^{\mu}$ [25],

$$
\begin{aligned}
\dot{\pi}^{\langle\mu \nu\rangle}+\frac{\pi^{\mu \nu}}{\tau_{\pi}} & =2 \beta_{\pi} \sigma^{\mu \nu}+2 \pi_{\gamma}^{\langle\mu} \omega^{\nu \gamma \gamma}-\frac{4}{3} \pi^{\mu \nu} \theta-\frac{10}{7} \pi_{\gamma}^{\langle\mu} \sigma^{v\rangle \gamma}, \\
\dot{n}^{\langle\mu\rangle}+\frac{n^{\mu}}{\tau_{n}} & =\beta_{n} \nabla^{\mu} \alpha-n_{v} \omega^{\nu \mu}-n^{\mu} \theta-\frac{3}{5} n_{v} \sigma^{\nu \mu}-\frac{3 \beta_{n}}{\epsilon+P} \pi^{\mu \nu} \nabla_{\nu} \alpha .
\end{aligned}
$$


Here $\omega^{\mu v} \equiv\left(\nabla^{\mu} u^{v}-\nabla^{v} u^{\mu}\right) / 2$ is the anti-symmetric vorticity tensor. It is important to note that in the relaxation-time approximation, $\tau_{R}$ is the common time scale for evolution of both $\pi^{\mu \nu}$ and $n^{\mu}$, i.e., $\tau_{\pi}=\tau_{n}=\tau_{R}$. Comparing the first-order equations, Eq. (15), with the relativistic Navier-Stokes equations, $\pi^{\mu \nu}=2 \eta \sigma^{\mu \nu}$ and $n^{\mu}=\kappa_{n} \nabla^{\mu} \alpha$, the dissipative relaxation times are given by $\tau_{\pi}=\eta / \beta_{\pi}$ and $\tau_{n}=\kappa_{n} / \beta_{n}$. It is interesting to note that for dissipative charge current evolution, the coefficients of $\Delta_{v}^{\mu} \partial_{\gamma} \pi^{\nu \gamma}$ and $\pi^{\mu \nu} \dot{u}_{v}$ vanish and hence they do not appear in Eq. (19).

The last term in Eq. (19) couples the evolution of dissipative charge current with that of the shear stress tensor. As shown in Ref. [29], this type of coupling leads to disagreement with transport results. However, we observe that using Eq. (15), the last term in Eq. (19) is equivalent to $-(6 / 5) n_{v} \sigma^{\nu \mu}$ up to second-order in the gradient expansion. Therefore the evolution equation, Eq. (19), for the dissipative charge current becomes [25]

$$
\dot{n}^{\langle\mu\rangle}+\frac{n^{\mu}}{\tau_{n}}=\beta_{n} \nabla^{\mu} \alpha-n_{\nu} \omega^{\nu \mu}-n^{\mu} \theta-\frac{9}{5} n_{\nu} \sigma^{\nu \mu}
$$

This is one of the main results of the present calculation. The compact form of the above equation makes it straightforward to directly implement it in a dissipative hydrodynamic code.

\section{Transport coefficients}

A measure of the relative importance of charge conductivity and shear viscosity is the dimensionless ratio $\kappa_{n} T / \eta$. Here, this quantity is given by $\kappa_{n} T / \eta=\beta_{n} T / \beta_{\pi}$, which is, in general, a function of $\mu / T$. In order to quantify this ratio, one still need to specify the appropriate degeneracy factors for quarks $\left(g_{q}\right)$ and gluons $\left(g_{g}\right)$, as

$$
\begin{aligned}
& g_{q}=N_{s} \times N_{c} \times N_{f}=6 N_{f}, \\
& g_{g}=N_{s} \times\left(N_{c}^{2}-1\right)=16,
\end{aligned}
$$

where $N_{c}=3$ is the number of colours, $N_{s}=2$ is the number of spin degrees of freedom, and $N_{f}$ is the number of flavours. In Fig. 1 (a), we show the ratio $\kappa_{n} T / \eta$ as a function of $\mu / T$ for $N_{f}=2$ and $N_{f}=3$. We see that, while this ratio is almost constant for small $\mu / T$, it drops rapidly for larger $\mu / T$, indicating that at low temperature and high density the conductivity of the QGP is small relative to the shear viscosity. Moreover, we observe that although the qualitative behaviour is the same for $N_{f}=2$ and $N_{f}=3$, the drop in $\kappa_{n} T / \eta$ is more pronounced for $N_{f}=3$. Furthermore, we note that for $\mu / T>1$ the ratio $\kappa_{n} T / \eta$ is almost independent of $N_{f}$.

Another interesting quantity is the ratio of heat conductivity to shear viscosity. Note that the heat flow is related to the dissipative charge current via the relation $q^{\mu}=-(\epsilon+P) n^{\mu} / n$, and hence we get [30]

$$
q^{\mu}=-\kappa_{q} \frac{n T^{2}}{\epsilon+P} \nabla^{\mu} \alpha \Rightarrow \kappa_{q}=\kappa_{n}\left(\frac{\epsilon+P}{n T}\right)^{2} \Rightarrow \frac{\kappa_{q}}{\eta}=\frac{\beta_{n}}{\beta_{\pi}}\left(\frac{\epsilon+P}{n T}\right)^{2},
$$

where $\kappa_{q}$ is the coefficient of heat conductivity. In Fig. 1 (b), we plot $\kappa_{q} / \eta$ scaled by the factor $\mu^{2} / \pi^{2} T$ against $\mu / T$, for a two and three flavor QGP. We see a constant behaviour in the limit of small as well as large $\mu / T$. Moreover, we see that $\left(\kappa_{q} / \eta\right) \mu^{2} / \pi^{2} T$ is independent of number of flavors for large $\mu / T$. These limiting behaviours have interesting consequences.

In the limit of both small and large $\mu / T$, the last equality in Eq. (22) reduces to [25]

$$
\frac{\kappa_{q}}{\eta}=C \frac{\pi^{2} T}{\mu^{2}}
$$



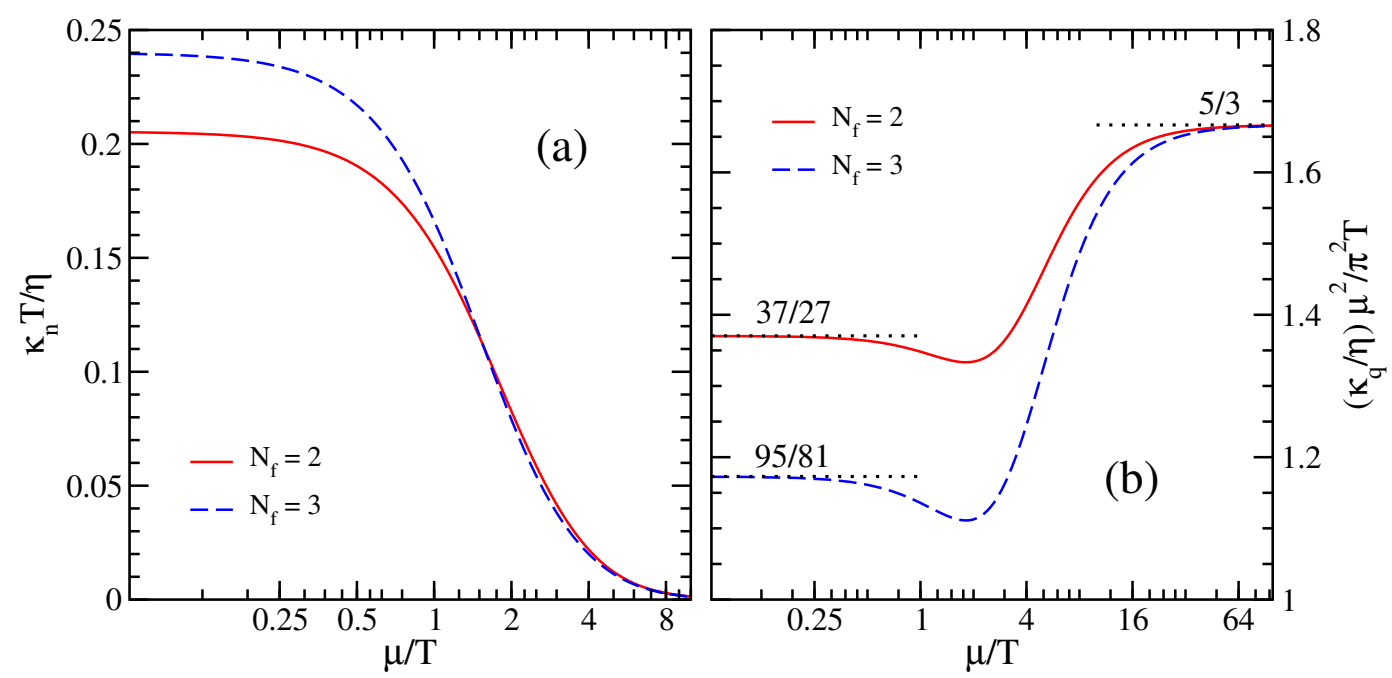

Figure 1. (a): The ratio of charge conductivity to shear viscosity scaled by the temperature, $\kappa_{n} T / \eta$, and (b): The ratio of thermal conductivity to shear viscosity, $\kappa_{q} / \eta$, scaled by the factor $\mu^{2} / \pi^{2} T$, for two flavor (solid line) and three flavor (dashed line) massless quarks, plotted against $\mu / T$.

which is analogous to the Wiedemann-Franz law [30]. The factor $\pi^{2}$ in the above equation is entirely due to quantum statistics and does not appear for a classical Boltzmann gas. In the small $\mu / T$ limit, the constant $C$ in Eq. (23) becomes $C=\left(4 g_{g}+7 g_{q}\right) / 9 g_{q}$. Hence, $C=37 / 27$ and $95 / 81$ for two and three flavor QGP, respectively; see Fig. 1 (b). On the other hand, in the large $\mu / T$ limit, we find $C=5 / 3$, independent of the number of flavors, as shown in Fig. 1 (b). It is interesting to note that these values of $C$ are comparable to $C=8 / 9$ (here a factor of $1 / 9$ indicates that the quark chemical potential is one-third of the baryon chemical potential) obtained in the calculations for strongly coupled conformal fluid with finite chemical potential [30]. Indeed, it is intriguing that up to a constant of proportionality, the limiting behaviour of the ratio $\kappa_{q} / \eta$ obtained here is identical to that derived in the case of a strongly coupled conformal plasma.

\section{Conclusions}

In this work we employed the iterative Chapman-Enskog method to solve the Boltzmann equation and then derived the second-order dissipative hydrodynamical equations for a system of massless quarks and gluons. For such a system the bulk viscous pressure vanishes and therefore the dissipation is solely due to shear stress tensor and dissipative charge current. We considered quantum statistics with non vanishing quark chemical potential for the equilibrium distribution function. We obtained novel, exact expressions for the second-order transport coefficients corresponding to the dissipative charge current evolution. We also demonstrated that the evolution equations for dissipative charge current and shear stress tensor can be decoupled. We found that, when the ratio of chemical potential to temperature is large, the charge conductivity is small compared to the shear viscosity. Finally, we showed that the limiting behaviour of the ratio of heat conductivity to shear viscosity is qualitatively identical to that of a conformal fluid in the strong coupling regime. 
Acknowledgements:- A.J. thanks Gabriel Denicol for useful discussions. A.J. was supported by the Frankfurt Institute for Advanced Studies (FIAS). The work of B.F. was supported in part by the Extreme Matter Institute EMMI. K.R. acknowledges support by the Polish Science Foundation (NCN), under Maestro grant DEC-2013/10/A/ST2/00106, and of the U.S. Department of Energy under Grant No. DE-FG02- 05ER41367.

\section{References}

[1] P. Romatschke and U. Romatschke, Phys. Rev. Lett. 99, 172301 (2007).

[2] H. Song and U. W. Heinz, Phys. Rev. C 77, 064901 (2008).

[3] M. Luzum and P. Romatschke, Phys. Rev. Lett. 103, 262302 (2009).

[4] B. Schenke, S. Jeon and C. Gale, Phys. Rev. Lett. 106, 042301 (2011).

[5] H. Song, S. A. Bass, U. Heinz, T. Hirano and C. Shen, Phys. Rev. Lett. 106, 192301 (2011); 109, 139904(E) (2012).

[6] R. S. Bhalerao, A. Jaiswal and S. Pal, Phys. Rev. C 92, 014903 (2015).

[7] A. Jaiswal and V. Koch, arXiv:1508.05878 [nucl-th].

[8] U. Heinz and R. Snellings, Ann. Rev. Nucl. Part. Sci. 63, 123 (2013).

[9] H. Grad, Comm. Pure Appl. Math. 2, 331 (1949).

[10] I. Muller, Z. Phys. 198, 329 (1967).

[11] W. Israel and J. M. Stewart, Annals Phys. 118, 341 (1979).

[12] G. S. Denicol, T. Koide and D. H. Rischke, Phys. Rev. Lett. 105, 162501 (2010).

[13] A. Jaiswal, R. S. Bhalerao and S. Pal, Phys. Rev. C 87, 021901(R) (2013).

[14] A. Jaiswal, R. S. Bhalerao and S. Pal, Phys. Lett. B 720, 347 (2013); J. Phys. Conf. Ser. 422, 012003 (2013); arXiv:1303.1892 [nucl-th].

[15] A. El, Z. Xu and C. Greiner, Phys. Rev. C 81, 041901 (2010).

[16] G. S. Denicol, H. Niemi, E. Molnar and D. H. Rischke, Phys. Rev. D 85, 114047 (2012).

[17] P. Romatschke, Phys. Rev. D 85, 065012 (2012).

[18] A. Jaiswal, Phys. Rev. C 87, 051901(R) (2013); arXiv:1408.0867 [nucl-th].

[19] A. Jaiswal, Phys. Rev. C 88, 021903(R) (2013); Nucl. Phys. A 931, 1205 (2014); Proc. Indian Natl. Sci. Acad. 81, no. 1, 62 (2015).

[20] C. Chattopadhyay, A. Jaiswal, S. Pal and R. Ryblewski, Phys. Rev. C 91, 024917 (2015).

[21] A. Jaiswal, R. Ryblewski and M. Strickland, Phys. Rev. C 90, 044908 (2014).

[22] W. Florkowski, A. Jaiswal, E. Maksymiuk, R. Ryblewski and M. Strickland, Phys. Rev. C 91, 054907 (2015).

[23] R. S. Bhalerao, A. Jaiswal, S. Pal and V. Sreekanth, Phys. Rev. C 88, 044911 (2013).

[24] R. S. Bhalerao, A. Jaiswal, S. Pal and V. Sreekanth, Phys. Rev. C 89, 054903 (2014).

[25] A. Jaiswal, B. Friman and K. Redlich, Phys. Lett. B 751, 548 (2015).

[26] S. Chapman and T. G. Cowling, The Mathematical Theory of Non-uniform Gases (Cambridge

University Press, Cambridge, 1970), 3rd ed.

[27] R. Vogt, Ultrarelativistic Heavy-Ion Collisions, (North-Holland, Amsterdam, 2007).

[28] J. L. Anderson and H. R. Witting Physica 74, 466 (1974).

[29] I. Bouras, E. Molnar, H. Niemi, Z. Xu, A. El, O. Fochler, C. Greiner and D. H. Rischke, Phys.

Rev. C 82, 024910 (2010).

[30] D. T. Son and A. O. Starinets, JHEP 0603, 052 (2006). 